\title{
A new synthesis of 5-substituted-3-phenyl-2H-pyrano[2,3-b]quinolin-2-ones
}

\author{
N. Venkatesh Kumar, B. Subramani and S. P. Rajendran* \\ Department of Chemistry. Bharathiar University, Coimbatore-641 (046, India
}

Manuscript received 17 May 2002, accepted 23 May 2003

Synthesis of title compounds and derivatives is reported by the Perkin reaction of 3-formyl-4-phenyl/methyl-2-quinolones (3) with sodium salt of phenylacetic acid. The 3-formyl-2-quinolones 3 were obtained from 2-chloro-3-formyl-4-phenyl/ methylquinolines (2) which inturn were prepared from 2-chloro-4-phenyl/methyl-3-vinylquinolines (1).

The synthesis of pyranoquinolines has gained momentum in recent years as they constitute parent ring structure of pyranoquinoline alkaloids which have been reported to be associated with interesting pharmacological properties ${ }^{\text {. }}$. biological and chemical properties ${ }^{2}$. Herein we report a convenient and new methodology for the synthesis of 2 chloro-3-formyl-4-phenyl/methylquinolines from 2-chloro4-phenyl/methyl-3-vinylquinolines as precursors and to utilise them for the construction of 5-phenyl/methyl-3-phenyl-2H-pyrano[2,3-b]quinolin-2-ones which are expected to be pharmacologically active (Scheme 1).

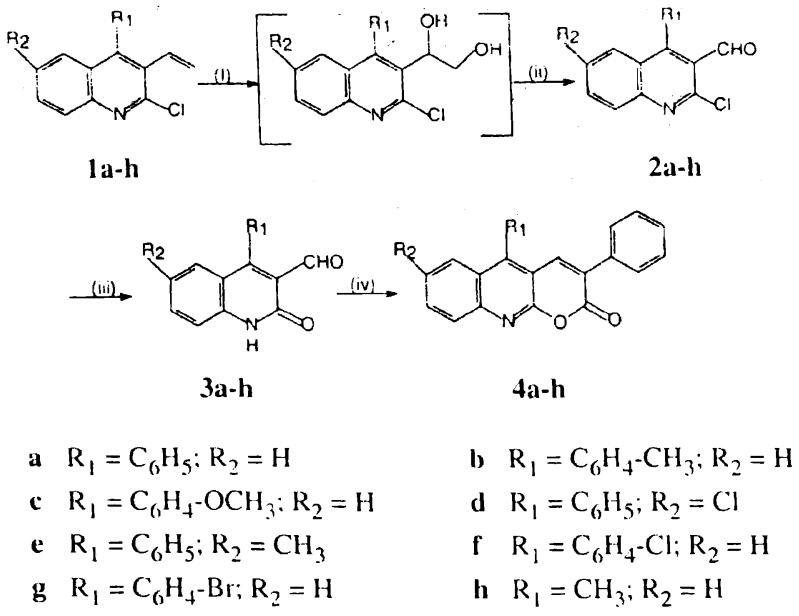

Scheme 1. Reagents : (i) $\mathrm{KMnO}_{4}$ /t-butanol, (ii) $\mathrm{CH}_{3} \mathrm{OH} / \mathrm{NaIO}_{4}$, (iii) $4 \mathrm{M} \mathrm{HCl}$, (iv) $\mathrm{C}_{6} \mathrm{H}_{5} \mathrm{CH}_{2} \mathrm{COONa} / \mathrm{Ac}_{2} \mathrm{O}$.

\section{Results and Discussion}

2-Amino benzophenone was treated with 3-butenoyl chloride to get 4-phenyl-3-vinyl-2-quinolone $\mathrm{e}^{3,4}$. This on heating with $\mathrm{POCl}_{3}$ produces 2-chloro-3-vinyl-4-phenylquinoline (1a). The chloroquinoline (1a) so obtained was treated with $\mathrm{KMnO}_{4}$ and $\mathrm{NaOH}$ at $0^{\circ}$ with constant stirring for 3-5 min in t-butanol medium and after passing $\mathrm{SO}_{2}$ gas, the resultant mass was continuously extracted with methylene chloride using Soxhlet apparatus to give a pasty mass.
The pasty mass was then dissolved in methanol and treated with $\mathrm{NaIO}_{4}$ at $3^{\circ}$ with stirring for $15 \mathrm{~min}$ and after adding $\mathrm{NaCl}$ the contents were extracted with chloroform gave 2chloro-3-formyl-4-phenylquinoline (2a) in $40 \%$ yield, m.p. $135-137^{\circ}$. Its IR spectrum showed peaks at $1705 \mathrm{~cm}^{-1}$ $(\mathrm{CHO})$ and $1080 \mathrm{~cm}^{-1}(\mathrm{C}-\mathrm{Cl})$. The chloroaldehyde (2a) so obtained was then converted to oxo compound (3a) by refluxing with aqueous $4 \mathrm{M} \mathrm{HCl}$ followed by usual work up gave a produce in $80 \%$ yicld, which melts at $302-304^{\circ}$. Its IR spectrum showed peaks at $1705 \mathrm{~cm}^{-1}$ (CHO) and 1620 $\mathrm{cm}^{-1}(-\mathrm{NHC}=\mathrm{O})$. The 3-formyl-4-phenyl-2-quinolone (3a) so obtained was subjected to Perkin reaction with sodium phenyl acetate and acetic anhydride gave a compound in $69 \%$ yield, which melts at $287-288^{\circ}$. From IR, ${ }^{1} \mathrm{H}$ NMR and mass spectrum data (Table 2), the compound was identified as 3,5-diphenyl-2H-pyrano|2,3-b|quinoline-2-one $(4 a)$. The reaction sequence leading to $4 a$ was then extended to synthesise $(\mathbf{4 b}-\mathbf{h})$. The compound (4h) was synthesised by following a similar procedure starting from 2-amino acelophenone through the intermediate 2-chloro-4-methyl3-vinyl quinoline' (1h).

\section{Experimental}

Melting points were determined using Raaga Melting Point apparatus and are uncorrected. IR spectra were recorded on a Perkin-Elmer 597 Infrared spectrometer as $\mathrm{KBr}$ pellets. ${ }^{1} \mathrm{H}$ NMR spectra were recorded on a AMX 40() spectrometer in $\mathrm{CDCl}_{3}$. Mass spectra were recorded on a Jeol-D 300 Mass spectrometer. Elemental analysis were performed by Perkin-Elmer model 24()B CHN analyser. For all the compounds satisfactory microanalysis values were obtained (C, $\mathrm{H}, \mathrm{N} \pm 0.4 \%)$

Typical procedures : 2-chloro-3-formyl-4-phenyll methylquinolines ${ }^{6,7}(2 \mathbf{a}-\mathbf{h}):$ A solution of $\mathrm{KMnO}_{4}(0.0) 148$ $\mathrm{mol})$ and $\mathrm{NaOH}(0.00125 \mathrm{~mol})$ in $8 \mathrm{ml}$ of water cooled to $0^{\circ}$, was added quickly with vigorous stiming to a freezing mixture of t-butanol ( $10 \mathrm{ml}), 2 \mathrm{ml}$ of water and 2-chloro-4phenyl/methyl-3-vinylquinoline ${ }^{3-5}(0.001 \mathrm{~mol})$. After 3-5 
min, most of the permanganate colour has been discharged and $\mathrm{SO}_{2}$ gas was passed immediately to ensure complete reduction of permanganate. The precipitate of $\mathrm{MnO}_{2}$ was filtered and most of the t-butanol was removed by distillation at atmospheric pressure and the resultant mass was continuously extracted with methylene chloride using Soxhlet apparatus for $48 \mathrm{~h}$. The compound obtained as a pasty mass while removing the solvent, was taken to subsequent experiment without purification. The pasty mass so obtained was dissolved in minimum amount of methanol and $\mathrm{NaIO}_{4}$ ( $0.5 \mathrm{mmol})$ in $3 \mathrm{ml}$ of water at $3^{\circ}$ was added dropwise with stirring. After 15 min a saturated solution of $\mathrm{NaCl}$ was added and the contents were extracted with chloroform. After evaporation the compound was separated as a yellow solid which was chromatographed over silica gel using petroleum ether-ethyl acetate mixture $(90: 10, v / v)$. The product was then recrystallized from chloroform (Table 1 ).

3-Formyl-4-phenl/methyl-2-quinolones ${ }^{8}$ (3a-h) : A mixture of $2(0.0104 \mathrm{~mol})$ and aqueous hydrochloric acid $(35 \mathrm{ml}: 4 \mathrm{M})$ was heated under reflux for $8 \mathrm{~h}$ in an oil bath at 12()$-130^{\circ}$ and then allowed to cool at room temperature. After $1 \mathrm{~h}$ the reaction mixture was poured into crushed ice, when the product was separated as a yellow solid. It was liltered, washed with water, dried and recrystallized from acpueous acetic acid (Table 1).

5-Phenyl/methyl-3-phenyl-2H-pyranol2,3-b/quinolin2 -ones' (4a-h) : A mixture of $3(0.001 \mathrm{~mol})$, freshly fused sodium phenyl acetate $(0.0() 16 \mathrm{~mol})$, acetic anhydride (0.05 mol) was heated under reflux for $8 \mathrm{~h}$ at $170-180^{\circ}$ in an oil

\begin{tabular}{|c|c|c|c|c|}
\hline \multicolumn{5}{|c|}{ Table 1. Physical and spectroscopic ditta of $\mathbf{2} \mathbf{a}-\mathbf{h}^{\prime \prime}$ and $\mathbf{3} \mathbf{a}-\mathbf{h}^{\prime \prime}$} \\
\hline Compd. & $\begin{array}{l}\text { M.p. } \\
{ }^{\circ} \mathrm{C}\end{array}$ & $\begin{array}{c}\text { Yield } \\
\%\end{array}$ & $\begin{array}{c}\mathrm{IR}^{\mathrm{c}} \\
\mathrm{cm} \mathrm{m}^{-1}\end{array}$ & $\begin{array}{c}\mathrm{MS} \mathrm{m/z} \\
\left(\mathrm{M}^{+}\right)\end{array}$ \\
\hline 2a & $135-1.37^{10}$ & 40 & $1705,2860,1080$ & $\begin{array}{l}267 \\
269\end{array}$ \\
\hline 2b) & $161-162^{10}$ & 41 & $1705,2870,1085$ & $\begin{array}{l}281 \\
28.3\end{array}$ \\
\hline $2 \mathrm{c}$ & $122-124^{10}$ & 40 & $1700,2864,1086$ & $\begin{array}{l}297 \\
299\end{array}$ \\
\hline $2 d$ & $168-170$ & 42 & $1695,2875,1080$ & $\begin{array}{l}302 \\
304\end{array}$ \\
\hline 2e & $129-131^{10}$ & 41 & $1710,2865,1090$ & $\begin{array}{l}281 \\
283\end{array}$ \\
\hline 21 & $143-145$ & 42 & $1705,2875.1076$ & $\begin{array}{l}302 \\
304\end{array}$ \\
\hline $2 g$ & $1.38-1.40$ & 40 & $1705,2862,1081$ & $\begin{array}{l}347 \\
349\end{array}$ \\
\hline $21 \mathbf{l}$ & $119-120$ & 40 & $1705.2950,1080$ & $\begin{array}{l}205 \\
207\end{array}$ \\
\hline 3a & $302-304$ & 80 & 1705.1620 & 249 \\
\hline $3 b$ & $308-310$ & 80 & 1705,1630 & 263 \\
\hline $3 c$ & $284-286$ & 79 & 1710,1625 & 299 \\
\hline $3 d$ & $320-321$ & 80 & 1710.1630 & $\begin{array}{l}284 \\
286\end{array}$ \\
\hline $3 e$ & $277-278$ & 80 & 1705,1620 & 263 \\
\hline 31 & $315-317$ & 79 & 1690.1610 & $\begin{array}{l}284 \\
286\end{array}$ \\
\hline $3 g$ & $311-313$ & 78 & 1710.1615 & $\begin{array}{l}329 \\
331\end{array}$ \\
\hline $3 h$ & $299-300$ & 80 & 1705,1640 & 187 \\
\hline
\end{tabular}

Table 2. Physical and spectroscopic data of $4 a-h^{\prime \prime}$

\begin{tabular}{|c|c|c|c|c|}
\hline Compel. & $\begin{array}{c}\text { M.p.o' } \\
\text { (Yicld \%) }\end{array}$ & $\begin{array}{c}\mathrm{IR}^{b} \\
\mathrm{~cm}^{-1}\end{array}$ & $\begin{array}{l}{ }^{\prime} H \text { NMR' } \\
(\delta) p p m\end{array}$ & $\begin{array}{l}\mathrm{MS} m /= \\
\left(\mathrm{M}^{+}\right)\end{array}$ \\
\hline ta & $\begin{array}{l}287-288 \\
(69)\end{array}$ & $\begin{array}{l}1749 \\
1050\end{array}$ & $\begin{array}{l}\delta 7.27-7.69\left(1+1 \mathrm{H}, \mathrm{m}, \mathrm{C}_{3} \text { and } \mathrm{C}_{5}-\mathrm{Ar}-\mathrm{H}, \mathrm{C}_{4}-\mathrm{H}\right. \\
\left.\mathrm{C}_{6}-\mathrm{H}, \mathrm{C}_{7}-\mathrm{H} \text { and } \mathrm{C}_{8}-1 \mathrm{H}\right) ; \delta 8.16\left(\mathrm{H}, \mathrm{d}, \mathrm{C}_{6}-\mathrm{H}\right)\end{array}$ & 349 \\
\hline th & $\begin{array}{c}295-296 \\
(70)\end{array}$ & $\begin{array}{l}1750 \\
1648\end{array}$ & 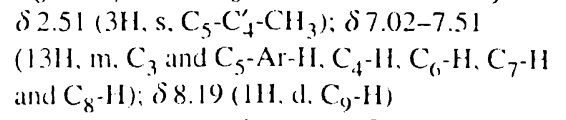 & 363 \\
\hline $4 c$ & $\begin{array}{c}271-272 \\
(70)\end{array}$ & $\begin{array}{l}1762 \\
1660\end{array}$ & $\begin{array}{l}\delta 3.64\left(3 \mathrm{H}, \mathrm{s}, \mathrm{C}_{5}-\mathrm{C}_{4}^{\prime}-\mathrm{OCH}_{3}\right) ; \delta 7.01-7.64 \\
\text { (13H, m. } \mathrm{C}_{3} \text { and } \mathrm{C}_{5}-\mathrm{Ar}-\mathrm{H}, \mathrm{C}_{4}-\mathrm{H}, \mathrm{C}_{6}-\mathrm{H}, \\
\left.\mathrm{C}_{7}-\mathrm{H} \text { and } \mathrm{C}_{8}-\mathrm{H}\right) ; \delta 8.19\left(1 \mathrm{H}, \mathrm{d}, \mathrm{C}_{6}-\mathrm{H}\right)\end{array}$ & 379 \\
\hline $4 d$ & $\begin{array}{l}297-298 \\
(70)\end{array}$ & $\begin{array}{l}1735 \\
1650\end{array}$ & $\begin{array}{l}\delta 7.39-7.73\left(1.3 \mathrm{H}, \mathrm{m}, \mathrm{C}_{3} \text { and } \mathrm{C}_{5}-\mathrm{Ar}-\mathrm{H}, \mathrm{C}_{4}-\mathrm{H},\right. \\
\left.\left.\mathrm{C}_{6},-11 \text { and } \mathrm{C}_{8}-\mathrm{H}\right) ; \delta 8.161 \mathrm{H}, \mathrm{d}, \mathrm{C}_{-9}-\mathrm{H}\right)\end{array}$ & $\begin{array}{l}384 \\
386\end{array}$ \\
\hline $4 e$ & $\begin{array}{c}282-284 \\
(70)\end{array}$ & $\begin{array}{l}1748 \\
1647\end{array}$ & $\begin{array}{l}\delta 2.62\left(3 \mathrm{H}, \mathrm{s}, \mathrm{C}_{7}-\mathrm{CH}_{3}\right): \delta 7.12-7.71(13 \mathrm{H}, \mathrm{m} \text {. } \\
\left.\mathrm{C}_{3} \text { and } \mathrm{C}_{5}-\mathrm{Ar}-\mathrm{H}, \mathrm{C}_{4}-\mathrm{H}, \mathrm{C}_{6}-\mathrm{H} \text { and } \mathrm{C}_{8}-\mathrm{H}\right) \text { : } \\
\delta 8.29\left(1 \mathrm{H}, \mathrm{d}, \mathrm{C}_{9}-\mathrm{H}\right)\end{array}$ & 363 \\
\hline $4 f$ & $\begin{array}{l}298-299 \\
(65)\end{array}$ & $\begin{array}{l}1760 \\
16.55\end{array}$ & $\begin{array}{l}\delta 7.21-7.8 .31\left(1.3 \mathrm{H}, \mathrm{m}, \mathrm{C}_{3} \text { and } \mathrm{C}_{5}-\mathrm{Ar}_{-} \mathrm{H}, \mathrm{C}_{4}-\mathrm{H} .\right. \\
\left.\mathrm{C}_{6}-\mathrm{H}, \mathrm{C}_{7}-\mathrm{H} \text { and } \mathrm{C}_{8}-\mathrm{H}\right) ; \delta 8.14\left(1 \mathrm{H}, \mathrm{d}, \mathrm{C}_{6}-\mathrm{H}\right)\end{array}$ & $\begin{array}{l}384 \\
386\end{array}$ \\
\hline $4 g$ & $\begin{array}{l}292(d) \\
(6.5)\end{array}$ & $\begin{array}{l}1755 \\
1050\end{array}$ & $\begin{array}{l}\delta 7.36-7.86\left(1.3 \mathrm{H}, \mathrm{m}, \mathrm{C}_{3} \text { and } \mathrm{C}_{5}-\mathrm{Ar}-\mathrm{H}, \mathrm{C}_{4}-\mathrm{H}\right. \\
\left.\mathrm{C}_{6}-\mathrm{H}, \mathrm{C}_{7}-\mathrm{H} \text { and } \mathrm{C}_{8}-\mathrm{H}\right) ; \delta 8.29\left(1 \mathrm{H}, \mathrm{d}, \mathrm{C}_{9}-\mathrm{H}\right)\end{array}$ & $\begin{array}{l}429 \\
431\end{array}$ \\
\hline 4h & $\begin{array}{c}285(d) \\
(70)\end{array}$ & $\begin{array}{l}1720 \\
1625\end{array}$ & & 287 \\
\hline
\end{tabular}




\section{J. Indian Chem. Soc., Vol. 80, October 2003}

bath. The reaction mixture was then poured into crushed ice $(\approx 100 \mathrm{~g})$ with stirring and kept aside for $4-5 \mathrm{~h}$. The resulting solid was filtered, dried and recrystallized from acetic acid to yield $\mathbf{4 a - h}$ (Table 2).

\section{References}

1. H. T. Openshaw in the 'Alkaloids'. ed. R. H. F. Manske. Academic Press, New York, 1967, Vol. IX, p. 223.

2. D. Lednieer and L. A. Mitscher, "Organic Chemistry of Drug Synthesis". Wiley, New York, 1977, Vol. I, p. 330.

3. P. Shanmugam, R. Palaniappan, N. Soundararajan, T. K. Thiruvengadam and K. Kanakarajan, Monatsch. Chem. 1976, 107, 259.
4. M. Murugesan, K. Ramasamy and P. Shanmugam, $Z$. Naturforsch., 1980, 35b, 746.

5. P. Shanmugam, P. Lakshminarayana and R. Palaniappan, Monatsch. Chem., 1973, 104, 633.

6. Kenneth B. Wilberg and Klaus A. Saegebarth, J. Am. Chem. Soc., 1957, 79, 2822.

7. J. Foos, F. Stcel, S.W.A. Rizvi and G. Fraenkel, J. Org. Chem., 1979, 44, 2533.

8. T. Tilakaraj and S. Y. Ambekar, J. Indian Chem. Soc., 1985, 62, 251.

9. T. Tilakaraj and S. Y. Ambekar, J. Indian Chem. Soc., 1986, 63, 981. 